\title{
Effects of Group Performance Feedback and Goal-setting in an Organisational Energy Intervention
}

\author{
Derek Foster \\ School of Computer Science \\ University of Lincoln \\ Lincoln, LN6 7TS, UK \\ +441522837130 \\ defoster@lincoln.ac.uk
}

\author{
Conor Linehan \\ School of Computer Science \\ University of Lincoln \\ Lincoln, LN6 7TS, UK \\ +441522837084 \\ clinehan@lincoln.ac.uk
}

\author{
Shaun Lawson \\ School of Computer Science \\ University of Lincoln \\ Lincoln, LN6 7TS, UK \\ +441522886318 \\ slawson@lincoln.ac.uk
}

\begin{abstract}
End-user energy demand (EUED) in the workplace is affected by a complex interaction between behavioural, social, technological, regulatory and organisational factors. Designing technology-led interventions to encourage pro-environmental behaviour that acknowledge and support this complexity is a significant challenge. This paper discusses the design and evaluation of an EUED intervention implemented in the corporate infrastructure of a UK university administration department. Two intervention types, group feedback and group goal-setting were implemented. 16 participants were recruited and engaged with a four stage study (baseline, group feedback, group goal setting, and baseline) for a duration of 4 months. This study design allowed us to track clearly any changes in mid-term energy usage behaviour during and beyond intervention. Findings suggest that, surprisingly, participant energy consumption increased during the intervention period compared to baseline conditions. These results demonstrate that simple group-based behaviour change methods can be counter-productive in the workplace, illustrating the complex and unpredictable nature of intervention in this design space.
\end{abstract}

\section{Categories and Subject Descriptors}

H.5.m. Information interfaces and presentation (e.g., HCI):

\section{General Terms}

Design; Human Factors.

\section{Keywords}

Sustainability; behaviour change; organisations.

\section{INTRODUCTION}

EUED in large organisations and small-medium enterprises (SME's) accounts for a significant proportion of a nation's energy requirements. In Europe, the services sector saw a 30\% growth in EUED in the period 1990-2009, with computers and other appliances in the office substantially contributing to this [8]. In the UK, for example, $10 \%$ of total energy consumed in 2011-2012 was within the services sector, which accounts for services and

Permission to make digital or hard copies of all or part of this work for personal or classroom use is granted without fee provided that copies are not made or distributed for profit or commercial advantage and that copies bear this notice and the full citation on the first page. Copyrights for components of this work owned by others than ACM must be honored. Abstracting with credit is permitted. To copy otherwise, or republish, to post on servers or to redistribute to lists, requires prior specific permission and/or a fee. Request permissions from Permissions@acm.org.

AcademicMindTrek '14, November 04 - 07 2014, Tampere, Finland

Copyright 2014 ACM 978-1-4503-3006-0/14/11 ...\$15.00

http://dx.doi.org/10.1145/2676467.2676481 business such as retail, hotel and catering, education, and agriculture [9]. Governments and utility providers view rising energy consumption as a serious issue in terms of carbon emissions and ability to deliver energy grid capacity to meet national demand. As a result governments, for example in the UK, have incentivised emission reductions through fiscal measures (carbon taxes) directly mapped to an organisations size and consumption practices [9],[24]. Research and grey literature around optimizing energy efficiency for large organisations and SME's largely focuses on automation (lighting and heating), equipment efficiency, and retrofitting [22],[13],[14],[31]. Although there are some exceptions, this literature has largely ignored the role of employees as end-users of energy in organisations. The design and rigorous validation of innovative approaches targeted at reducing EUED in organisations is therefore a topic requiring urgent attention from the HCI sustainability research community. Indeed, EUED within the workplace is no different to other organisational or design problems, in that it can be considered the result of the interplay between behavioural, social, technological, design, organisational, and regulatory factors.

This paper presents a study conducted in a workplace environment designed to promote reductions in EUED. The remainder of this paper is outlined as follows: i) a background discussion of HCI research in behaviour change for energy interventions, ii) intervention design and technical implementation, iii) experimental method description, iv) statistical and qualitative results, and finally v) discussion of findings.

\section{BACKGROUND}

\subsection{Designing Energy Interventions}

HCI researchers have produced a body of literature on the design of technologies that attempt to promote pro-environmental behaviour. A significant portion of the published work is focussed on the design of technology-led feedback interventions for the domestic space, for example see [10],[27],[2].

\subsubsection{Designing Feedback}

A comprehensive review on the design of eco-feedback technology was carried out by Froehlich et al. [12], who examined a corpus of papers generated by both the HCI and environmental psychology communities. They found that feedback [10] and rewards [4] were useful in engaging participants throughout an intervention, and in some cases reduced energy consumption levels. Design methods such as prototyping, participatory design, 
and focus groups were found useful in increasing participant engagement in interventions, and helped researchers to understand the complexity of the challenge in this design space. However, the authors indicated that further work is required by the $\mathrm{HCI}$ community to understand how to best evaluate the effectiveness of feedback technology on longer-term energy consumption.

\subsubsection{Evaluating Feedback}

Notably, Froehlich et al. highlighted shortcomings in evaluation methodologies in the HCI literature, in which user studies averaged at 2.5 weeks in duration, compared with 15.5 months in work reported by environmental psychologists. Crucially, without longitudinal findings for behaviour change studies there is no way to validate that a particular HCI method or feedback design is effective to change behaviour. Technologies designed by the HCI community for changing behaviour would benefit from evaluation through evidence based behaviour-change methods, over psychologically significant time scales, to bypass novelty effects [25], [1].

\subsection{Feedback interventions in the workplace}

The majority of HCI research on the design of technology for promoting sustainability has been aimed at the domestic environment. Very little previous work has investigated whether end-user energy interventions could be useful in organisational contexts. The workplace presents a very different design space from the domestic, where end-users (i.e., employees), work under regulatory and organisational rules and are not responsible for paying the energy bill. These features outline a very different design space than that targeted by domestic energy interventions. However, some relevant recent work has been published, notably by Lockton et al., who investigate employee engagement with energy interventions [18]. Findings by Lockton et al. highlight that feedback for near-real time energy use, and rewards in the form of points helped to engage users. However, the authors found that energy consumption was not correlated with engagement levels.

Schwartz et al. present findings on a series of participatory design workshops, followed-up by the deployment of smart meters and energy usage visualisations in an organisation [28], with results suggesting that participants preferred feedback that visualised consumption related to individual devices, and generated by individual users. Embryonic work by Lehrer \& Vasude [16] investigated design concepts for using online social networks to deliver workplace energy feedback. They have yet to deploy a field study using the produced designs. Work in progress by Milenkovic et al. report the outcomes of an attempt to engage office workers with personalised energy feedback [20], with the novel ability for users to provide feedback on their own comfort levels in an office or building. Early results by Milenkovic et al. show their approach can provide a more holistic view of consumption in a building for management purposes, and also increase reflection on awareness of energy use by employees. In summary, HCI sustainability research in the workplace has been somewhat limited in scope, and the design space is only beginning to be understood.

For some time, work in the environmental psychology domain by Siero et al. [29] remained the only rigorous research carried out in energy-related behaviour-change interventions in organisations. The work demonstrated the effectiveness of group comparative feedback to reduce energy consumption, indicating that future technology-enabled studies could build upon and adopt the same approach. Very recent work by Murtagh et al. investigated the effectiveness of individual energy feedback in an office to reduce energy use, with some success [21]. Findings of the Murtagh et al. study revealed reductions in some of the intervention phases, but also uncovered some issues with engagement, and inconsistencies with energy reductions across the intervention period, revealing the complexities of workplace interventions.

In order to adequately design and develop a workplace energy intervention using HCI methods, we draw upon previous research [11], which undertook a rigorous qualitative study involving 65 employees from a number of organisations to understand the design challenges for organisational energy interventions. The work produced a framework of key themes detailing user perceptions and energy intervention design considerations, with some of the considerations echoing grey literature produced for energy efficiency strategies in businesses [31]. The findings provided a scaffolding for the design of successful workplace interventions, with each theme representing an abstracted intervention component. The themes are now briefly described.

\subsubsection{Incentives}

Suggestions for implementing incentives as part of energy interventions were disparate, and ranged from small rewards, such as free meals, to sweeping high impact, high cost rewards such as employing more staff. Whatever the reward, participants suggested it must be highly visible, with frequent progress updates. Incentives were closely related to the engagement theme.

\subsubsection{Engagement}

Competition and negotiated targets (goal-setting) in an intervention were favoured to both lower barriers to participation and to engage with an intervention; with particular emphasis on achievable targets and goals. Unrealistic targets, inability to set targets, and unfair competition were highly cited for bringing about potential disengagement.

\subsubsection{Openness}

Trust and privacy were seen as important issues for employee engagement with energy interventions. For the intervention to be engaging, employees would have to accept the reasons why savings were required without being cynical. Having confidence that the organisation was acting in the employee's best interest was a key theme. Participant privacy, in the context of presenting only group feedback as opposed to individual, was cited as being important to preserve anonymity.

\subsubsection{Leadership role}

Without "management commitment" employees feared their efforts in an intervention would be frustrated, that results would be trivial and interventions destined to fail. Charismatic leadership and leading change from the top were cited as being the most motivating factors in engaging with and adhering to interventions, with change "trickling down".

\subsubsection{Communication}

This was seen as critical at all stages of an intervention. Communication encompassed workshops for educating employees on energy as a finite resource, effective marketing campaigns for recruitment, and continuous feedback using multiple channels for all aspects of energy use in the organisation. Quality of the message, not the mode of communication, was seen as the important factor. 


\subsubsection{Visualisation}

Representation of energy i.e., bar graphs and other abstract visualisations is a contentious issue. It is dependent on the technically feasible and organisational policies in the context of energy data ownership. There was a preference for bar charts, line graphs and metrics such as cost; typical organisation-centric representations

\subsection{Motivating Behaviour Change}

The previous section described the absence of evaluation strategies in the HCI literature appropriate for evaluating the long term effectiveness of workplace energy interventions. Significantly, the design of digitally mediated energy interventions reported in the HCI literature is also lacking in solid foundation in behavioural science, highlighting the complexities of mapping design to behaviour theory. However, some HCI papers have recently acknowledged this problem and are exploring social \& behavioural psychology frameworks [1],[12]. The science of behaviour modification (now referred to as applied behaviour analysis) spans decades of research [5]. It defines rigorous evidence based methods for intervention through quantification and controlled experimentation, and is immediately and practically useful for anyone designing technology-mediated behavioural interventions [17]. For example, the psychology research suggests a combination of regular feedback and realistic goal-setting may prove a useful approach in promoting behaviour change. In the study reported in this paper, we implement feedback through the design of energy usage visualisations, and apply goal setting through weekly group-based goal setting tasks [5],[19]. The group contingencies implemented are based solidly in those reported in the behaviour modification literature, and are discussed in detail later in this work.

A prototype application was trialled across 4 phases (baseline, group feedback, group goal setting, and baseline) over a 4 month period with 16 participants in an Estates and Facilities (EF) department. Being an organisational intervention, it targeted the individual employee as well as the collective, through a common goal (i.e., energy reduction). A mixed methods approach allowed us to accurately measure energy use, as well as offering supporting explanations on how energy was consumed through participant practices. Our quantitative research questions were:

- Does group-based feedback reduce energy consumption?

- Does group goal-setting reduce energy consumption?

- Do engagement levels change with each condition?

- What are the interface preferences for energy feedback?

Additionally, we posed qualitative questions through interviews with findings positioned to provide a rich account of participants' reflections on engaging with the study. Example questions were i) "Overall, do you feel the office used more or less energy as a group during the study?" and ii) "Could you discuss which parts of the study you felt were the most effective in terms of engagement?".

\section{DESIGN}

In an ideal scenario, a workplace intervention would aim to include all of the previously discussed design themes (see [11] for an in-depth discussion). However, in this study we were constrained by the policies of the organisation with whom we were working. Specifically, we did not have sufficient management buy-in to implement the incentives theme. We were not able to reward employees financially for reductions observed in energy consumption. However, rather than a limitation of our study, this constraint demonstrates the realistic and valid challenges faced when designing technology-mediated energy interventions for organisations. Indeed, lack of buy-in from people in leadership roles was identified in previous work as potentially detrimental to employee engagement with any workplace intervention. Participants previously expressed concern over; 'where do any energy savings go?', and 'is there any management buy-in? if not then why should we take part?' Despite this, we implemented the other five themes; communication, engagement, leadership role, openness, and visualisation.

The final design of the intervention widget interfaces were based on sketching and paper-prototyping produced during previously reported workshops [11]. It's important to note that simple numeric illustrations and graphs were desired. Intervention aspects of design considerations are explained in more detail in sections $3.1-3.3$

\subsection{Baseline}

To measure baseline energy consumption (non-intervention) an energy appliance monitor was installed for each participant $(n=16)$, to monitor total energy consumption at their desk space once every minute. Energy data was sent wirelessly to a basestation which relayed it to a database server and an Internet of Things storage platform. Baseline data was collected for a period of 1 month before and after the intervention phases. Additionally, an online questionnaire was developed and distributed via email to all staff working in the department before the study started. The questionnaire aimed to elicit basic responses in how employees perceive and consume energy in workplace practices. Interviews were also carried out on study completion to help unpick how participants engaged with the intervention and influenced the findings. Due to limited space and scope, this work presents findings from the interviews over questionnaire responses.

\subsection{Group Feedback}

The first stage of the intervention delivered energy consumption feedback at the group, rather than individual, level. This decision was primarily made because of concerns expressed over openness and visualisation themes during prototyping workshops [11]. Participants requested energy consumption feedback be delivered at the group level, rather than the individual, because of worries about how these data could be used by others to draw inferences about how they do their job.

In designing 'group-based' interventions, we were heavily influenced by the work of Siero et al. [29], who designed energy feedback mechanisms appropriate for groups of employees in an organisation setting. Siero et al. focussed on bringing about collective behaviour change in a group using comparative feedback by providing performance feedback on other groups and comparing it to own group. Indeed, behavioural psychology research, more generally, has shown that group contingencies, when properly managed, can bring about effective results [5].

The dependent group contingency method [5] was selected as it allows a whole group to share a positive reward, even if it is just an individual or a small number of users within the group whose performance is responsible for reducing their energy consumption. In other words this type of group contingency means consequences are delivered to the entire group based on the performance of one participant, or a subset of the larger group. This directly supports goal attainment feedback as discussed in 
the next section. Group feedback was delivered for 6 weeks. Crucially, the first two weeks data in this condition was removed from the data set analysed, due to the potential for novelty effects.

\subsection{Group Goal Setting}

The second stage of the intervention again delivered energy consumption feedback at the group level, and also allowed for the collective setting of weekly goals for reductions in energy consumption. Group goal-setting was designed to support the communication, engagement, and visualisation design themes (see [11]). Goals were designed that were public, provided progress feedback, and had a completion deadline [19]. At the start of each working week, when in the goal setting condition, participants were prompted to view the widget and select an optional energy saving goal. Participants were able to set a savings goal between $1-5 \%$ of the total energy used in the previous week. Participants indicated their desired goal target individually, and a final goal for that week was calculated based on an average of all goals submitted by participants. The group goal-setting condition was delivered for 6 weeks, again with the first two weeks removed from data analysis for potential novelty effects. Feedback was displayed to participants on goal progress, with final goal positive/negative attainment feedback displayed at the end of the working week.

\section{IMPLEMENTATION}

A number of technical challenges were addressed to deploy the intervention widget to participant's desktop computers. In order to drive engagement and limit the complexity of interacting with the widget, we deployed it as part of the corporate network domain. This meant when each participant logged onto their computer with their domain credentials, the widget would automatically load on their desktop. This required senior-management buy-in at the highest level and was a positive step forward for intervention engagement, it also addressed the requirement of the leadershiprole theme for intervention design [11].

When initially logged in the widget would move first through a 'loading screen', then the 'main screen', with a number of supporting screens available to click through for further information, energy tips, and switching the feedback view between fiscal and $\mathrm{kWh}$ units.

A detailed illustration of the widget's features are shown in figure 1. The loading screen imparts feedback and energy saving tips (comparing work to home energy use) and information on whether or not the group is saving energy compared to the same time period last week, with the main screen displaying total energy used in the current weekly time period, updated automatically every minute.

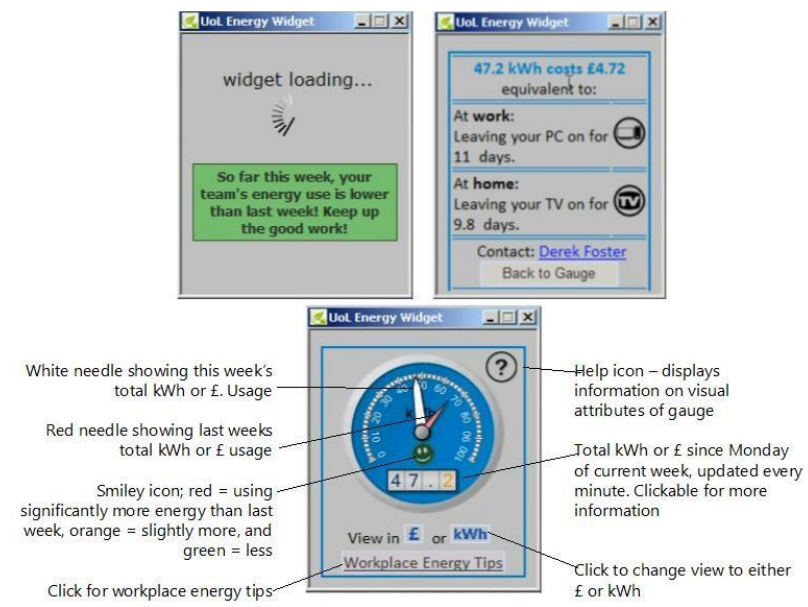

Figure 1. Intervention widget in feedback condition

To accurately measure each participant's energy use we configured each desk's electrical power sockets in such a way that they mapped onto an individual participants desk area. This ensured we could obtain an aggregate energy reading for all electrical appliances a participant used. Participants had a great deal of control over the number and type of equipment and devices they could utilise at their desk space; for example portable heaters, multiple chargers, secondary computers such as laptops, desk lights, and even rechargeable power tools were all evident on site visits.

Appliance monitoring devices [6] measured each desk's total energy consumption every 60 seconds for transmitting wirelessly to a base station. Custom software was developed to store the energy data and expose it for consumption through a public REST API on Internet of Things storage platform Xively [32]. Approximately $3.5 \mathrm{~m}$ energy and interaction data-points were generated for analysis. See figure 2 for technical diagram.

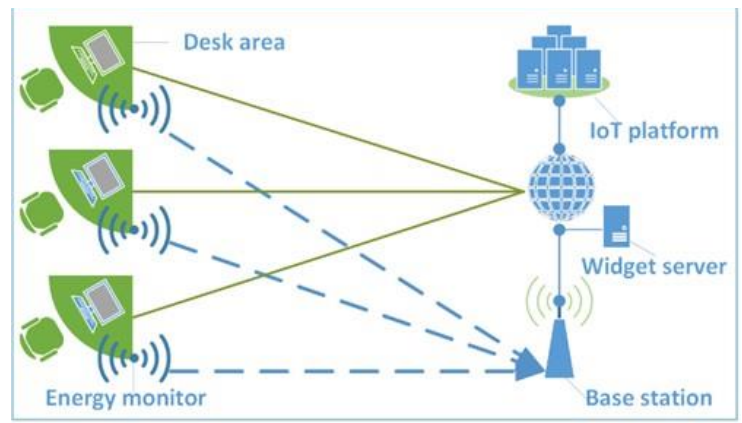

Figure 2. Technical implementation of widget

\section{METHOD}

Deployment of the intervention field study featured staff as participants solely from an EF administration department in a UK university. In a UK context, EF departments handle the management of physical environments to support staff and students.

The staff all worked in a large open-plan office with diverse roles including engineers, space development, and residential services. The aim of the study was to observe if the intervention phases had an effect on the EF department group's energy consumption. 


\subsection{Participants}

Sixteen employees were recruited as participants to trial the widget, 5 female. They were comprised of a diverse range of roles including engineers, general admin, space management, building control, and from various levels of organisational management. All participants used a work-supplied computer connected to the corporate network domain. It was clearly communicated that they were free to opt-out of the study at any time without question.

\subsection{Design}

The field trial study followed a single-subjects A-B-A design commonly used in the behavioural sciences to evaluate the effects of intervention upon a behaviour of interest (see [5]). A baseline condition was used to determine an expected level and variance of the behaviour of interest. Two intervention conditions (group feedback and goal setting) were introduced consecutively with the intention of impacting upon that behaviour. A post-study baseline was used to judge whether any observed changes in behaviour during intervention were stable, generalisable and long-term, or whether behaviour returned to baseline levels when intervention conditions were removed.

To clarify, the independent variable had four conditions; baseline, group feedback, group goal-setting, and baseline. The main dependent variable was the energy used in $\mathrm{kWh}$ for each condition, with other dependent variables to measure engagement and interaction levels. In the group feedback condition participants could view the group's energy consumption in near real-time, and compare it to the previous weeks total. In the group goal-setting condition participants' could suggest an energy savings goal, with progress towards the goal displayed. In both conditions energy tips could be viewed, example tips were "...put computer in standby mode when away for short periods.", and "...turn off portable appliances and chargers when not in use."

In order to address the likelihood of intervention conditions producing a novelty or halo effect [3] when taking part in a study and presented with new technology, we decided to remove the first two weeks of energy data from each condition in our analysis. This was also a requirement for generating equal time epochs for meaningful descriptive and inferential analysis to be made.

As well as eliminating the first two weeks of energy data, we also removed weekend data, as staff were not normally contracted to work over the weekend period. This gave us a final dataset that covered a 4 month period, with 20 days per month for MondayFriday, producing 80 days of energy consumption data.

\section{RESULTS}

The main metric of measurement was $\mathrm{kWh}$, calculated from snapshots of watt-seconds once per minute, using the formula '(power * 60) / 3600000' to calculate kWh. To put the energy use into context, the average daily total consumption by a participant across each of the study phases was: pre-study $=1.0517 \mathrm{kWh}$, feedback condition $=1.1846 \mathrm{kWh}$, goal-setting condition = $1.1894 \mathrm{kWh}$, and post-study $=1,155 \mathrm{kWh}$. During the baseline pre-study phase, this equated to around $5.08 \mathrm{kWh}$ consumed by each participant during Monday-Friday. Comparatively, 5.08kWh is approximately half the daily energy use of the average UK household [23]. The descriptive data clearly indicates participants used more energy in the intervention conditions. The absolute total consumption in $\mathrm{kWh}$ for each month from the raw data was month 1: 336.5 , month 2: 378.9 , month 3: 380.5 , and month 4: 356.8. Figure 3 illustrates the daily total average use for participants, with the mean shown for each month of the study, while a sample of participants $(n=5)$ was used to graph absolute daily consumption totals over the entire study in figure 4 .

\subsection{Statistical Significance}

With the descriptive results indicating more energy was used in the intervention conditions over the baseline measurement, we performed a repeated measures ANOVA to check for significance in the findings. The daily absolute values for total $\mathrm{kWh}$ used by each participant were used as the input data for the ANOVA analysis. Results indicated assumptions of sphericity had been violated $\chi^{2}(5)=30.42, p=0.00$, which we corrected using the Greenhouse-Geisser estimates of sphericity $(\varepsilon=.94)$. The results of the ANOVA show that the effects of the intervention were significant $F(2.81,894.85)=3.16, p=0.27$. Drilling down further using a pairwise comparison, we found pre-study vs. feedback ( $p$ $=0.034)$ and pre-study vs. goal-setting $(p=0.023)$ were significant. However, the conditions: feedback vs. goal-setting ( $p$ $=1$ ) and pre-study vs. post-study $(p=1)$ were found to be nonsignificant.

Although the data indicated we could discard the null hypothesis of feedback and goal-setting conditions having no effect over prestudy measurements, we were presented with the fact that participant energy had actually increased by statistically significant levels. In other words the intervention conditions appear to have had the opposite of the intended effect of reducing energy consumption. Qualitative data to support unpicking this interesting finding is presented in the interview data later in this section, with further examination in the main discussion section.

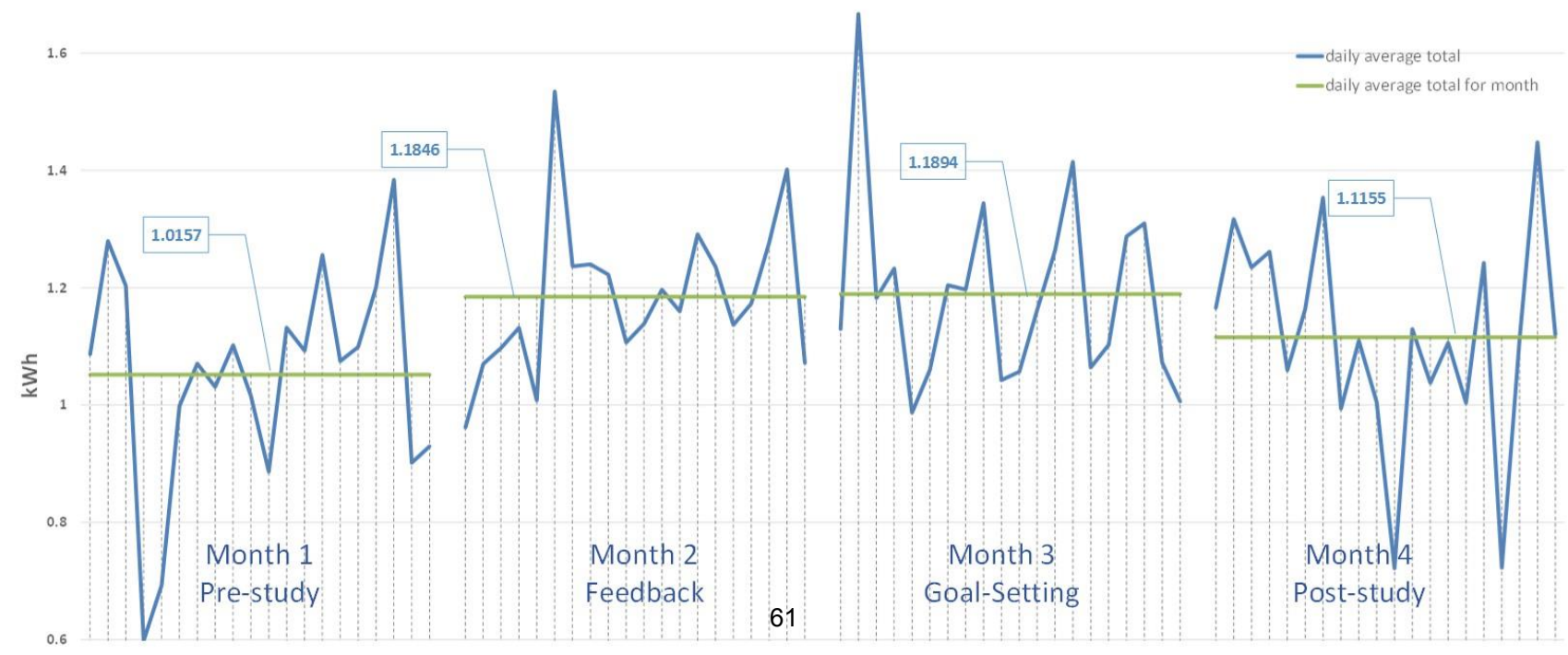

Figure 3. Total daily average participant consumption across all phases of study 


\subsection{Engagement Levels}

In order for interventions to be successful, adequate engagement is required. Engagement and interaction with the energy widget was tracked covering: 1) duration widget displayed during working day and energy tip viewing, 2) selection of interface type (kWh vs. fiscal), and finally 3 ) goal setting activity.

\subsubsection{Widget viewing and energy tips}

To understand daily use of the widget when it initially loads and whether it is left running on the desktop or simply closed by a participant, we recorded the interaction data. Overall there were relatively high levels of engagement, with the widget displayed for an average of 7.7 hours for each participant in the feedback condition, and 6 hours a day in the goal-setting condition. For viewing energy tips there was little uptake, which essentially provided comparative information on the current weekly energy total. A total of 44 energy tips views were recorded from 8 participants.

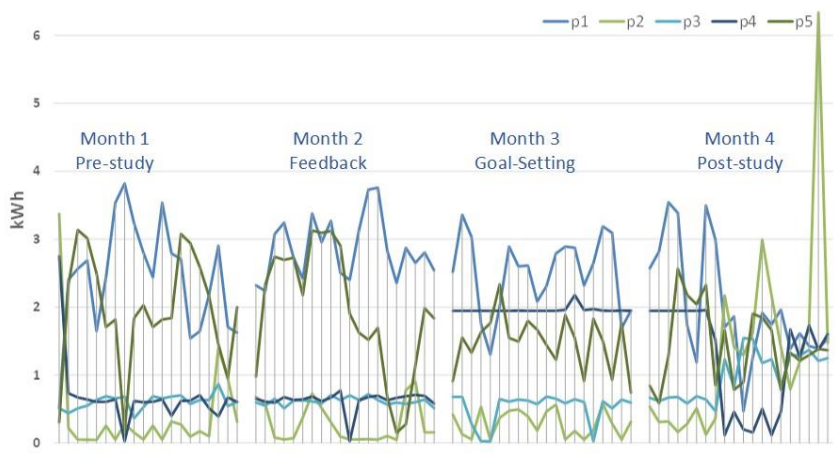

Figure 4. Sample participants' daily energy use in each month

\subsubsection{Choice of $k$ Wh or fiscal units}

The widget provided the functionality to swap between $\mathrm{kWh}$ and fiscal units, allowing participants to choose the interface they preferred. The default selection was $\mathrm{kWh}$ with very little deviation, however 11 participants did try the fiscal view over a few days.

\subsubsection{Goal setting}

Four group goal-setting events were carried out in the goalsetting condition, with goal activity recorded including data on goal achievement. Table 1 shows that two goals were met (goals 1 and 4), with the remaining two goals using more energy in the previous week. Encouragingly the number of participants taking part in goal-setting never fell below $50 \%$, given that goal-setting was optional. Engagement with goal setting was fairly positive with at least $50 \%$ of participants submitting a target savings goal.

Table 1. Goal-setting engagement

\begin{tabular}{|l|c|c|c|}
\hline & Participants & Target Goal & Saving \\
\hline Goal 1 & 10 & $3.87 \mathrm{kWh}, 4 \%$ & $14 \%$ \\
\hline Goal 2 & 8 & $1.67 \mathrm{kWh}, 2 \%$ & $-7 \%$ \\
\hline Goal 3 & 9 & $2.80 \mathrm{kWh}, 3 \%$ & $-2 \%$ \\
\hline Goal 4 & 11 & $2.86 \mathrm{kWh}, 3 \%$ & $3 \%$ \\
\hline
\end{tabular}

In summary, the descriptive and inferential statistics show participants used significantly more energy in the intervention feedback and goal-setting conditions when compared to the baseline pre-study measurement. Post-study measures indicated energy consumption fell to near pre-study levels, in other words it was a return to baseline measures, indicating there was a clear impact during the group feedback and group goal-setting conditions. Overall engagement levels with the widget were high.

\subsection{Interview Data}

To provide a richer, and more insightful narrative on participant engagement with the study over and above energy use alone, we conducted post-study interviews with 6 participants. The interview data presented here supports unpicking what happened during the intervention phases, with data analysed using a smallscale grounded theory approach [30]. The first two stages of grounded theory were utilised, namely open-coding and axialcoding. Axial coding provides the emergent themes from grouping and categorizing open codes. Each theme offers an interpretation of the data to give insight, and to help explain attitudes and actions by participants during the study. Four themes emerged from the data: i) Non-negotiable practices; ii) Technology constraints; iii) Efficiency awareness; and iv) Circumvention. Each theme is now discussed in turn with supporting conversational segments.

\subsubsection{Non-negotiable practices}

This theme focused on the dissonance between pro-environmental behaviour and carrying out necessary activities specific to a job role P1: "It is my view that the reasons behaviours do not change is on the basis of our job role practices being more deeply ingrained, like job priorities and habits.", and $\mathrm{P} 4$ : "I didn't really change my pattern of work during the study as I had my usual stuff to do". These comments suggest work routine conflicted with action to make energy savings.

\subsubsection{Technology constraints}

Participants were vocal in describing how current technologies they use at work impede making reductions: P2: "I felt that there was no way to minimise the energy used due to the current way that remote access works at the university i.e., you have to have your PC on all the time you wish to work remotely", and P1: "new equipment was installed (such as the new Cisco video phones which have replaced the already over-egged display phones we had. I just need a simple phone, preferably one powered from the phone line, not with its own transformer using unnecessary electricity!)". Technology constraints highlight lack of employee control and ownership of equipment used at work, linked to employees normally being excluded from the procurement process. In support of P2's claim of remote working requiring a PC be switched on constantly, we identified power signatures in the data consistent with this.

\subsubsection{Efficiency awareness}

This theme highlighted divisive feelings on the usefulness of widget energy tips. Some felt they were not useful - P5: "I rated tips as the least effective because this is basic information that all people already know", and P1: "I didn't look at the energy tips as I already do all I can", while others were more positive, P6: "The feedback and energy tips were useful. Also just having the widget generated discussion in the office about energy saving.", and P3: "I do think it will have encouraged a few colleagues to be more energy aware and perhaps switch off more often than before".

\subsubsection{Circumvention}

The field study was located in a single department over a large floor space. The area incorporated automated controls for lighting and heating with no way to override them locally. However 
suggestions were made that some participants were using portable heaters and lighting at their desk space - P1: "some of my colleagues use personal heaters in the morning at their desk on colder days", and P4: "the new LED lighting isn't very nice, its an unnatural kind of light so we sometimes bring in desk lights". It can be reasonably assumed that circumventing automated controls can lead to higher consumption overall. Indeed, P1's claim of portable heaters being deployed for personal use is supported in the energy data, with short energy spikes identified early morning at some of the desk spaces.

To summarise, the derived themes are linked in that for the most part they offer a series of 'organisational-led' reasons from participants in how they perceived savings could not be made. These findings propose potential explanations for the energy use evidenced in the intervention phases. However given the scope of our qualitative evaluation, and the complexities of employee work demands, we cannot easily draw out conclusive findings, but rather offer a reflection upon further design implications of such interventions. This is discussed, along with alternative experimental design methods, in the next section.

\section{DISCUSSION \& CONCLUSION}

It is generally accepted that feedback is a useful tool to bring about reductions in energy use. For example, work by Darby [7] found that reductions of up to $15 \%$ are possible when feedback is displayed frequently. The findings of the current study, where energy use significantly increased over baseline levels during each intervention condition, do not reflect those of Darby.

Notably, the experimental design used in the current study, allowing for analysis of 'return to baseline' was, in the context of HCI sustainability research, uniquely suited to detect stable changes in participant behaviour. 'Return to baseline' is a concept commonly used in behavioural science to evaluate the effectiveness of an intervention (see [5]). In this method, an intervention is removed and the effects upon behaviour are observed. If behaviour remains stable despite the removal of intervention contingencies, we can infer that the participant has learned, and that the new behaviour is stable and has generalised. However, if the behaviour returns to the level recorded at pre intervention baseline, we can infer that the intervention itself was maintaining that behaviour and that no long term learning, or change in behaviour, has occurred. We found a clear return to baseline effect in this study. Thus, while goal-setting and feedback did not have the intended effect of lowering consumption, it did have a psychologically significant short-term effect on energy use behaviour.

Importantly, the findings of this study do not necessarily mean that a group-based approach to feedback and goal-setting is ineffective to reduce energy consumption in workplaces. Rather, it is likely that a more subtle implementation of a group contingency would be more effective. Our findings suggest that dependent group contingencies [5], which were used in the current study, are not an appropriate means for delivering group feedback and goalsetting in this context. However this intervention design is the simplest possible type of group intervention available. We decided that it was prudent to use the simplest possible intervention design in our first study on this topic.

A more complex interdependent group contingency [5] may be more suitable design for future studies [15]. Specifically, dependent group contingencies allow all group participants to share the success of receiving positive nominal feedback and achieving goals, even if the success is attributed to an individual or a small group of the larger group. In the case of the interdependent group contingency approach, all group members need to meet the criteria of reducing their own consumption, as well as that of the group goal, when compared to the previous week's performance. This enforces participants to work together to achieve a common goal, in this case a reduction in energy use. An applied example of an interdependent group contingency study was carried out by Poplin \& Skinner [26] with success. Of course, manipulation of the intervention design is a logical stepwise process, and we have learned through this study that dependent contingencies are not appropriate in this context.

We must also take into account the participant experience in order to uncover more complex issues that can hinder intervention success. Crucially, for any energy intervention to achieve a degree of success there has to be adequate levels of participant engagement. We found relatively high levels of engagement with the intervention widget being displayed for a significant part of the working day. There was no significant diminishment of engagement with feedback over time with an average of 7.7 hours and 6 hours of daily widget viewing time in the feedback and goal-setting conditions respectively. Goal-setting activity was also carried out by at least $50 \%$ of participants. In no way do the results suggest any disengagement took place that may contribute to higher consumption levels.

Other similar research to this study by Murtagh et al. [21] carried out a 4 month study that used an individual self-comparative feedback approach, with ambient feedback and temporal (to within an hour) graphs. They found inconsistencies with energy reductions with levels decreasing only in the $3^{\text {rd }}$ a $4^{\text {th }}$ months, despite no change in the intervention condition in months 1-4.

Perhaps the most striking similarity with this work and that of Murtagh et al. is the recurring element of employees offering explanations on why energy savings can't be made, a phenomena Murtagh et al. termed 'a syndrome of reasons'. For the most part, the reasons offered were shifted away from the employee and focussed instead on the organisational context. In other words responsibility to make savings appeared to be shifted from the individual and onto the organisational entity. This finding further compounds the difficulties in designing appropriate interventions in this design space, and highlights the complex relationship between feedback and behaviour in organisations. Indeed, in addition to the aforementioned issues, a number of external confounding factors may influence a workplace energy intervention such as staffing levels and seasonal weather effects. As such it is difficult to draw all-encompassing conclusions on best practice application of behaviour change methods for feedback and goal setting in a work place intervention. Even more so when very little rigorous work has been carried out to date. Rather, work should draw upon valuable qualitative accounts and empirical behaviour modification research and adopt an iterative approach in the stepwise implementation of selected behaviour change methods.

Findings from our qualitative analysis offer more granular design implications that could support the main design themes from previous work on designing organisational interventions [11]. The themes also offer possible explanations on the increased consumption evidenced in intervention phases of the study, and what might be avoided in future work. However, given the evidence of this study and previous relevant work, the unpredictable nature of such interventions remains a challenging 
obstacle. Careful consideration of experimental design, coupled with robust design considerations paves the way forward.

In summary, while the intervention employed in this study did not have the intended effect of lowering participant energy consumption, the advantage of basing the design and evaluation on established methodologies is clear; we have demonstrated that the simple intervention trialled here is not appropriate in this particular context, and based on our understanding of the behavioural science literature and findings in this study, we have a clear plan for moving forward, based on the implementation of more nuanced group feedback methodologies that has been demonstrated as effective in other, similar, contexts.

\section{REFERENCES}

[1] Ai He, H., Greenberg, S., and Huang, E.M. One size does not fit all: applying the transtheoretical model to energy feedback technology design. In Proc. CHI 2010, ACM Press (2010), 927-936.

[2] Chetty, M., Trana, D. and Grinter, R. Getting to green: understanding resource consumption in the home. In Proc. UbiComp 2008, ACM Press (2008), 242-251.

[3] Clark, R. E., and Sugrue, B. M. Research on instructional media, 1978-1988. In Educational media and technology yearbook, 14 (1988), 19-36.

[4] Bang, M., Gustafsson, A., and Katzeff, C. Promoting new patterns in household energy consumption with pervasive learning games. In Proc. Persuasive'07, Springer-Verlag (2007), 55-63.

[5] Cooper, J.O, Heron, T.E, and Heward, W.L. Applied Behaviour Analysis. Pearson, New Jersey, 2007.

[6] Current Cost Ltd. (2013) Current Cost - Reducing your energy bills so you can live a greener life!, http://www.currentcost.com/product-iams.html

[7] Darby, S. The effectiveness of feedback on energy consumption: A review for DEFRA of the literature on metering, billing and direct displays, Environmental Change Institute, University of Oxford, Oxford, 2006.

[8] EEA (2013) Final Energy Consumption by Sector. European Environment Agency, http://www.eea.europa.eu/data-and-maps/indicators/finalenergy-consumption-by-sector-5

[9] Environmental Agency UK (2013) CRC Energy Efficiency Scheme guidance, http://publications.environmentagency.gov.uk/PDF/GEHO0312BWGE-E-E.pdf

[10] Fischer, C. Feedback on household electricity consumption: a tool for saving energy? Energy Efficiency 1, 1, (2008), 79104.

[11] Foster, D., Lawson, S., Wardman, J., Blythe, M., and Linehan, C. "Watts in it for me?": design implications for implementing effective energy interventions in organisations. In Proc. CHI 2012, ACM Press (2012), 23572366.

[12] Froehlich, J., Findlater, L. and Landay, J. The design of ecofeedback technology. In Proc. CHI 2010, ACM Press (2010), 1999-2008.
[13] GreenBiz.com (2009) Green Teams - Engaging Employees in Sustainability, http://www.greenbiz.com/sites/default/files/GreenBizReport s-GreenTeams-final.pdf

[14] Intel (2009) Reducing Energy Use in Offices to Increase ITSustainability,,http://download.intel.com/it/pdf/Reducing Energy_Use_In_Offices.pdf

[15] Kerr, M. M., and Nelson, C. M. Strategies for addressing behaviour problems in the classroom $4^{\text {th }} E d$. (1988) New Jersey, Prentice Hall.

[16] Lehrer, D., and Vasudev, J. Evaluating a Social Media Application for Sustainability in the Workplace. In Proc. CHI 2011, ACM Press (2011), 2161-2166.

[17] Linehan, C., Kirman, B., and Roche, B. (2014, in press) Gamification as applied behaviour analysis. In Steffen P. Walz and Sebastian Deterding (eds) The Gameful World: Approaches, Issues, Applications. MIT Press. (2014 in press).

[18] Lockton, D., Cain, R., Harrison, D., and Nicholson, L. 'CarbonCulture at DECC: Digital Engagement for Sustainability at Work'. In Proc. HCI 2013, (2013).

[19] Martin, G., and Pear, J. Behaviour Modification: What it is and how to do it. Pearson, Boston.

[20] Milenkovic, M., Hanebutte, U., Huang, Y., Prendergast, D., and Pham, H. (2013) Improving user comfort and office energy efficiency with POEM (personal office energy monitor). In Ext. Abstracts CHI 2013, ACM Press (2013), $1455-1460$.

[21] Murtagh, N.,Nati, M., Headley, W.R., Gatersleben, B., Gluhak, A., Imran, M.A, and Uzzell, D. Individual energy use and feedback in an office setting: A field trial. In Journal of Energy Policy, ISSN 0301-4215.

[22] NEEFUSA (2009) The Engaged Organisation, online athttp://www.neefusa.org/BusinessEnv/EngagedOrganizatio n_03182009.pdf

[23] Ofgem (2011) Typical domestic energy consumption figures, online at https://www.ofgem.gov.uk/ofgempublications/76112/domestic-energy-consump-fig-fs.pdf

[24] Pearce, D. The political economy of an energy tax: The United Kingdom's Climate Change Levy. In Energy Economics 28, 2, (2006), 149-158.

[25] Prochaska, J.O. and DiClemente, C.C. Transtheoretical therapy: Toward a more integrative model of change. In Psychotherapy: Theory, Research \& Practice, Vol 19(3), (1982), 276-288.

[26] Poplin, J., and Skinner, C. Enhancing academic performance in a classroom serving students with serious emotional disturbance: Interdependent group contingencies with randomly selected components. In School Psychology Review 32, (2003), 282-296.

[27] Riche, Y., Dodge, J., and Metoyer., R.A. Studying alwayson electricity feedback in the home. In Proc. CHI 2010 ACM Press (2010), 1995-1998.

[28] Schwartz, T., Betz, M., Ramirez, L., and Stevens, G. Sustainable energy practices at work: understanding the role 
of workers in energy conservation. In Proc. NordiCHI 2010: ACM Press (2010), 452-462.

[29] Siero, F.W., Bakker, A.B, Dekker, and G.B, Van, M.T.C. Changing organizational energy consumption behavior through comparative feedback. In Journal of Environmental Psychology 16: (1996), 235-246.

[30] Strauss, A. Qualitative analysis for social scientists. Cambridge, Cambridge University Press, 1980.
[31] UK Cabinet Office (2011) Behaviour change and energy use,

https://www.gov.uk/government/publications/behaviourchange-and-energy-use-behavioural-insights-team-paper

[32] Xively Logmein (2013) Xively - Public Cloud for the Internet of Things, online at https://xively.com 